\title{
Evolutionary Origin and Molecular Biology of the Melanoma-Inducing Oncogene of Xiphophorus
}

\author{
ANGELIKA SCHARTL, NICOLA DIMITRIJEVIC, AND MANFRED SCHARTL \\ Biozentrum der Universität Würzburg, Physiologische Chemie I, Am Hubland, \\ D-97074 Würzburg, Germany
}

\begin{abstract}
Melanoma formation in platyfish/swordtail hybrids of genus Xiphophorus is due to overexpression of the receptor tyrosine kinase oncogene $\mathrm{Xmrk}$. This gene is the molecular equivalent to the $T u$-locus of platyfish, formerly identified by Mendelian genetics. The supposed evolutionary origin of the Xmrk oncogene is a nonhomologous recombination event in the $5^{\circ}$ region of the corresponding $\mathrm{X} m r k$ protooncogene with an anonymous sequence, $D$. This event led to a gene duplication of Xmrk, whereby the new copy obtained a novel promoter derived from $D$. Inactivity of this promoter in parental fish warrants lack of tumorigenicity of the $\mathrm{X} m r k$ oncogene in wild playfish. In hybrids, however, the promoter is active. This leads to the pigment cell transforming overexpression of Xmrk.
\end{abstract}

Key words: Receptor tyrosine kinase, Gene duplication, Macromelanophore, Modifier genes, Gene expression, Transgenic fish

\section{GENETIC CONTROL OF SPONTANEOUS MELANOMA FORMATION IN XIPHOPHORUS}

Fish of the genus Xiphophorus inhabit fresh water biotopes of the Atlantic drainage of Mexico, Honduras, and Guatemala. In several species, some individuals (ranging from 1 to $40 \%$ of a given population) exhibit spot patterns composed of large, intensely black pigment cells. These cells have been termed macromelanophores to distinguish them from micromelanophores, the normal-sized black pigment cells that make up the uniform greyish body coloration (Gordon, 1927). More than 60 years ago it was discovered that certain hybrids of macromelanophore pattern-carrying platyfish (Xiphophorus maculatus) and of unspotted swordtail ( $X$. helleri) spontaneously develop malignant melanoma (Gordon, 1927; Häussler, 1928; Kosswig, 1928).

Shortly thereafter it was recognized that occurrence of tumors in hybrids is dependent on a single locus, the macromelanophore locus of $X$. maculatus, which "interacted" with the $X$. helleri genome (Kosswig, 1929; Gordon, 1931). This interaction was later on defined as the effect of modifying genes. It has been a debate over the decades whether these modifying genes are "intensifiers" contributed by $X$. helleri to the hybrid offspring genome or if they are "suppressors," originally present in the $X$. maculatus genome, that are eliminated by the selective breeding process through substitution of the corresponding platyfish chromosomes by those from the swordtail (Gordon, 1958;
Atz, 1962; Kosswig, 1965; Zander, 1969; Kallman, 1970). Supposing multiple modifier genes melanoma formation was explained by the loss of "inhibitory" genes suppressing species-specifically the macromelanophore genes in the hybrid genome (Breider, 1952). The current, generally accepted explanation for the observed phenomena of hereditary melanoma by Anders and coworkers formalizes such considerations on the bases of numerous genetic experiments (Anders, 1990).

In a typical crossing experiment, a female $X$. maculatus that carries the X-chromosomal macromelanophore locus $S d$ (spotted dorsal, small spots in the dorsal fin) is mated to $X$. helleri, which does not carry the corresponding locus. The $F_{1}$ hybrids show enhancement of the $S d$ phenotype. Backcrossing of the $F_{1}$ hybrid to $X$. helleri results in offspring that segregate into $50 \%$ that have not inherited the $S d$-locus and are phenotypically like the $X$. helleri parental strain and $50 \%$ that carry the macromelanophore locus and develop melanoma. The severity of melanoma ranges from very benign in some individuals (phenotype like the $F_{1}$ hybrids) to highly malignant in others. Highly malignant melanomas of such fish grow invasive and

Address reprint requests to Prof. Dr. Manfred Schartl, Biocenter of the University of Würzburg, Physiological Chemistry I, Am Hubland, D-97074 Würzburg, Germany. 
exophytic and are fatal to the individuum. They even grow progressively following transplantation to thymusaplastic (nude) mice (Schartl and Peter, 1988).

Based on a variety of such classical crossing experiments, a genetic model has been developed to explain tumor formation in Xiphophorus (Ahuja and Anders, 1976). The macromelanophore locus was formally equated to a sex chromosomal multigene locus, whose critical constituent was designated "tumor-gene" $(T u)$. Melanoma formation then was attributed to the uncontrolled activity of $T u$. In non-tumorous fish $T u$ activity was proposed to be negatively controlled by cellular regulatory genes or tumor suppressor genes ( $R$-genes, corresponding to the repressing modifying genes mentioned above). In the crossing experiment outlined above this means that $X$. maculatus contains the $T u$-Sd locus on the X-chromosome and the corresponding major $R$ on an autosome, while $X$. helleri is proposed not to contain this particular $T u$-locus and its corresponding $R$. According to the model, backcrossing of the $T u$-containing hybrids to $X$. helleri results, in effect, in the progressive replacement of $R$-bearing chromosomes from $X$. maculatus by $R$-free chromosomes of $X$. helleri. The stepwise elimination of regulatory genes is thought to allow expression of the $T u$ phenotype, leading to benign pigment cell lesions if one functioral allele of $R$ is still present or malignant melanoma if $R$ is absent.

It is, however, similarly compatible with the crossing data to attribute $T u$ activity to the presence of intensifying genes contributed by $X$. helleri chromosomes to the hybrid genome, although this explanation has not been appreciated to the same extent as the suppressor hypothesis. To our knowledge, there is no crossing experiment that would help to decide between both possibilities.

Reintroduction of suppressor genes or diluting out activating genes, respectively, by crossing malignant melanoma bearing hybrids to parental $X$. maculatus was shown to lead to a reversion of the malignant phenotype resulting in totallytumor-free fish in the succeeding backcross generations using $X$. maculatus as the recurrent parent (Anders et al., 1984). This demonstrates that the melanoma oncogene $T u$ itself remains structurally unaltered during the process of activation via hybridization.

\section{THE Tu-LOCUS ENCODED Xmrk GENE}

Using strategies of positional cloning that were aided by precise knowledge of the chromosomal location of $T u$ and a plethora of chromosomal mutants affecting this region, a candidate gene was isolated that maps to the $T u$ locus (Schartl, 1988; Wittbrodt et al., 1989; Zechel et al., 1988). A full-length cDNA was isolated from a melanoma cell line (Wittbrodt et al., 1989). The cDNA sequence shows a highest similarity to the human epidermal growth factor receptor gene (HER). Because it was demonstrated that the isolated sequence is not the fish homolog of $\mathrm{HER}$ (Wittbrodt et al., 1989), it is consequently a novel member of subclass I of the multigene family of receptor tyrosine kinases (RTKs) and was designated X $m r k$, for Xiphophorus melanoma receptor kinase.

Two copies of Xmrk are located on the sex chromosomes, both of approximately $25-30 \mathrm{~kb}$. They show identical exon/ intron arrangements and exon sizes and match the genomic organization of higher vertebrate subclass I RTKs (Adam et al., 1991). One copy of Xmrk (INV) is invariably present in all Xiphophorus fish representing the protooncogenic version of $\mathrm{X} m r k$. The second copy is the oncogenic version of X $m r k$ and maps to the Tu-locus. It exists as X-and $\mathrm{Y}$ specific alleles. The INV-encoded $5.8 \mathrm{~kb}$ message is found at low levels in all tissues tested including melanoma (Mäueler et al., 1988; Wittbrodt et al., 1989; Dimitrijevic, unpublished). Furthermore, this transcript is differentially regulated during embryonic development (Wittbrodt et al., 1989). This implies a normal physiological function of INV and corroborates its identification as the protooncogene.

In contrast, the oncogenic mRNA of $4.7 \mathrm{~kb}$ is only expressed in melanoma but at very high levels. The level of transcription correlates with the degree of malignancy. In benign melanoma expression of the oncogene seems to be downregulated due to the activity of the modifying locus $R$ (Adam et al., 1991).

In contrast to the high degree of sequence conservation and structural similarity in the transcribed regions, the promoter of the Xmrk oncogene (Adam et al., 1993) diverges completely from the typical GC-rich promoters of genes belonging to the RTK family (Ishii et al., 1985). Unexpected structural elements like a TATA and CAAT box typical for "non-house-keeping" genes (Dynan, 1986) specify the Xmrk promoter. Sequence analysis of the Xmrk protooncogene cDNA isolated from a Xiphophorus embryonic cell line and comparison to the oncogenic version revealed overall sequence identity in the translated and $3^{\circ}$ untranslated region, but this terminates in the first exon (Adam et al., 1993). This led to the conclusion that the $5^{\prime}$ dissimilarity reflects a genomic breakpoint, i.e., the oncogenic and protooncogenic versions of $\mathrm{X} m r k$ must have different promoter regions. In Southern blots of Eco RI-digested DNA, a specific molecular probe from the oncogene promoter hybridized strongly to a $2.1-\mathrm{kb}$ band in addition to the oncogene promoter fragment. This signal was present in DNA of all fish tested regardless of the presence or absence of the $T u$-locus. The $2.1 \mathrm{~kb}$ band was cloned and shown to be almost identical in sequence to the oncogene promoter from its $5^{\prime}$ end to the transcription start site. There was no further sequence similarity $3^{\circ}$ of this point. This locus was called $D$ (for donor).

The sequence identity between the oncogene and the protooncogene over nearly the entire coding region on the one hand and the fact that the Xmrk oncogene and the $D$-locus share the same promoter region on the other hand gave rise to the following model for the origin of the Xmrk oncogene (Fig. 1). During evolution of Xiphophorus, an unprogrammed nonhomologous recombination event of the protooneogene with the D-locus has occurred. As a result the duplicated copy from the protooncogene obtained a novel $5^{\circ}$ region, namely the promoter region of the D-locus, but retained the coding part of the receptor tyrosine kinase gene (Adam et al., 1993). In consequence, the new chimeric gene locus is subjected to altered transcriptional regulation due to 

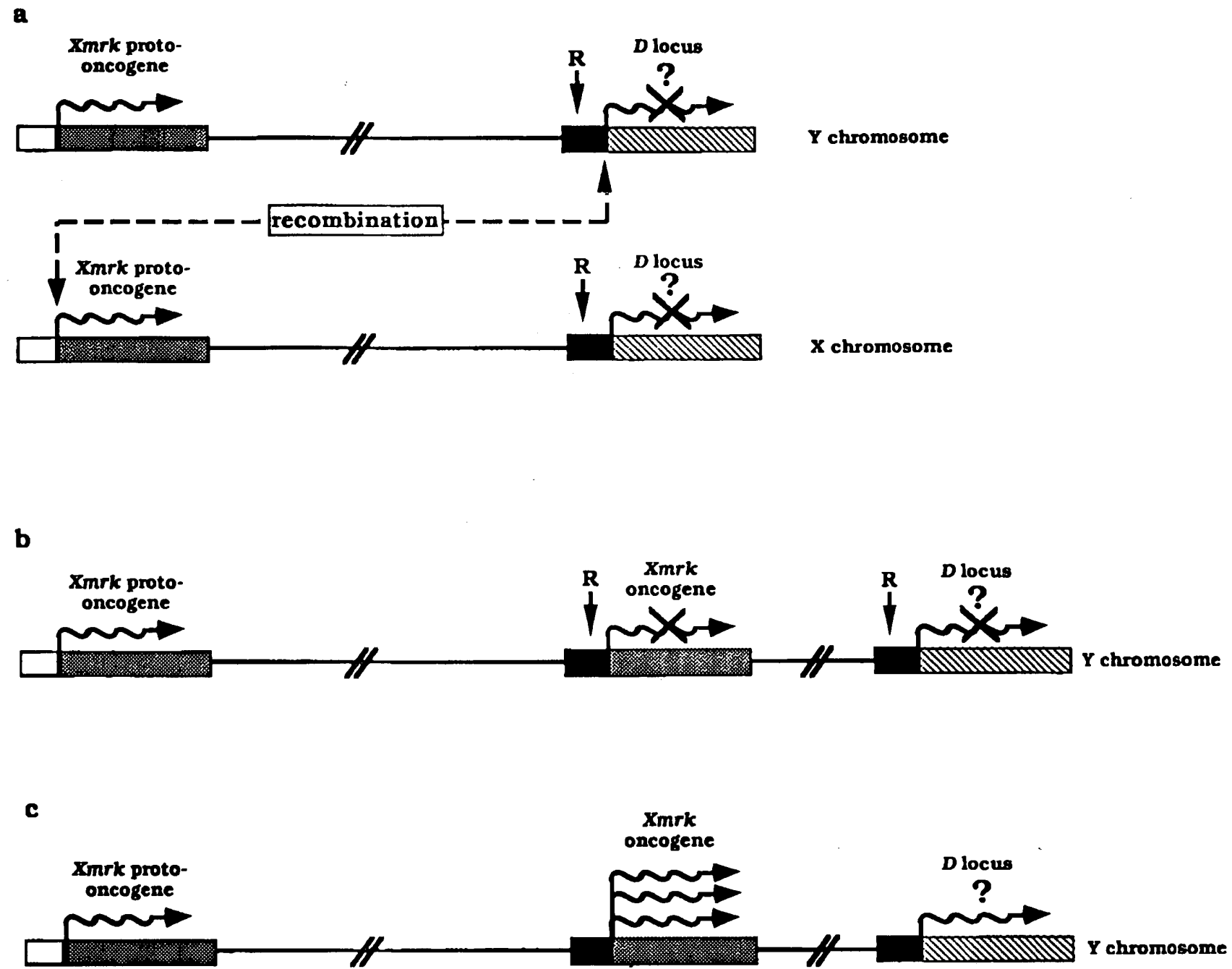

Fig. 1. Schematic representation of the genomic rearrangement leading to the $\mathrm{X} m r k$ oncogene. Rectangles represent genes. The dashed line indicates the recombination event. Regulation by the modifying locus $R$ is indicated assuming that $R$ acts as a suppressor of Tu. The promoter of the Xmrk protooncogene is white, the promoter of the $D$-locus is black. a: An unequal meiotic crossing over between the $\mathrm{X}$ and $\mathrm{Y}$ chromosome transfers the $\mathrm{X} m r k$ protooncogene to a new

its new promoter. While the oncogene promoter is quiet in wild-type fish due to the action of $R$, it becomes hyperactive in the hybrids as a result of the stepwise elimination of $\mathrm{R}$ by crossing. This leads to overexpression of the $\mathrm{X} m r k$ oncogene in pigment cells and to a nonphysiological overproduction of the $\mathrm{X} m r k$ gene product resulting in increased cell division and malignant melanoma. Until now, it has not been clear if unscheduled transcription of $\mathrm{X} m r k$ is the only reason for tumor formation, because comparison of the cDNAs of oncogene and protooncogene revealed some critical amino acid changes (Dimitrijevic, unpublished) that have to be tested functionally. Nevertheless, the change in transcriptional regulation seems to be the first and crucial step of melanogenesis. location on the $\mathrm{Y}$ chromosome, fusing it to the $D$ locus promoter. The truncated X chromosome is lost. b: Regulation of expression in wildtype $X$. maculatus. The $\mathrm{X} m r k$ oncogene is kept silent by $R$ via its new, $D$-derived promoter. c: Gene regulation in macromelanophores of hybrids lacking the $R$ locus due to crossing conditioned elimination. The $\mathrm{X} m r k$ oncogene is overexpressed and exerts its transforming function.

\section{THE Xmrk PROTEIN}

As predicted from the cDNA sequence, $\mathrm{X} m r k$ encodes a functional receptor tyrosine kinase. Experiments with chimeric receptors in which the extracellular domain of Xmrk was replaced by the corresponding part of the human EGF receptor $(\mathrm{HER})$ indicated that the Xmrk oncoprotein carries an activating mutation in its extracellular or transmembrane domain, which leads to a low level of constitutive autophosphorylation. Whether this feature contributes to the transforming activity of Xmrk is so far unknown.

The in-vitro transforming capability of Xmrk was shown by introducing the HER/mrk chimera in NIH 3T3 cells. After stimulation with EGF a high number of foci of transformed cells was observed comparable to the frequency 
after transformation with the viral $\operatorname{erb} B$ oncogene (Wittbrodt et al., 1992).

Xmrk is the most abundant phosphotyrosine protein in melanoma of Xiphophorus and thus is a highly activated growth factor receptor. Although the molecular structure of the ligand is still unknown, biochemical experiments have revealed that Xiphophorus melanoma cells secrete a protein factor that stimulates the Xmrk protein to become a highly active signal transducer (Malitschek et al., 1994). Thus the Xiphophorus melanoma cells are an autocrine and, thereby, growth autonomous system.

\section{$\mathrm{X} m r k$ IS $T u$}

All properties of Xmrk analyzed so far are in accordance with what can be expected for a dominant oncogene and its protein product. As with all other genes that have been cloned as "candidate genes", more evidence is expected to confirm that it is, indeed, the sought-after genetic entity responsible for the phenotype for which the locus has been assigned to, rather than just fulfilling a selection of criteria that theoretically could be met also by other genes or their products. The most stringent conditions to define $\mathrm{X} m r k$ as the $\mathrm{T} u$ gene are given by the question: Is $\mathrm{X} m r k$ necessary and sufficient for tumor formation? The first part was readily answered by analyzing the genomic organization of Xmrk in a mutant that had lost the capacity to develop melanoma. This mutant was found to be due to a large insertion in one exon in the kinase domain of Xmrk (Wittbrodt et al., 1989). The gene disruption of Xmrk abolishes the $T u$ phenotype, thereby proving that Xmrk is necessary for melanoma formation. For answering the second part of the question, transgenic fish were employed. An $\mathrm{X} m r k$ minigene was introduced into a closely related fish species, the Japanese Medakafish, Oryzias latipes, whichother than the live-bearing Xiphophorus-is egg-laying and therefore more suited for gene transfer experiments. Tumors appeared after a few days in the injected embryos (Winkler et al., 1994). The remarkably short latency period excludes the possibility that additional events, for instance mutations that lead to activation of host oncogenes or inactivation of tumor suppressor genes, were required for tumor formation in the $\mathrm{X} m r k$ transgenics. Thus $\mathrm{X} m r k$ alone is sufficient for tumor formation.

The transgenic experiments provided also other interesting information. For expression of the Xmrk transgene a promoter was used that is constitutively active in all tissues and organs of fish embryos. However tumors were detected only in those cell types in which the Xmrk protooncogene is found to be expressed (Winkler et al., 1994). This indicated that the $\mathrm{Xmrk}$ oncoprotein is dependent on a specific signal transducing machinery that acts downstream of the receptor to mediate its transforming capability.

\section{IS Xmrk THE MACROMEL $A$ NOPHORE GENE?}

Depending on the macromelanophore pattern of the parental platyfish, the melanoma of the hybrids are localized in specific body compartments, e.g., in crosses where the $S d$ locus that causes macromelanophore spots in the dor- sal fin of the parental platyfish was introduced into the hybrid genome, melanoma spread from the dorsal fin. In fish with an $S p$ (spotted) locus which causes macromelanophore spots on the flanks of the parental platyfish, melanoma of the corresponding hybrids originate exactly from this region. This pattern information and the neoplastic transformation locus Tu are extremely closely linked and only few mutants exist which affect the original macromelanophore pattern and, consequently, the melanoma compartment (Anders et al., 1973; Kallman, 1975). According to Kallman's work the different patterns are due to a series of alleles, or pseudoalleles, of macromelanophore genes each harboring the capacity for melanoma formation (Kallman, 1975), while Anders proposed that a single Tu-locus is closely linked to a series of nonallelic, compartment-specific genes that suppress appearance of macromelanophores in the various body regions. The different patterns then should be due to mutational impairment of one of the cis-acting compartment genes (Anders, 1991). The implication from both explanations is that the gene that determines the macromelanophore phenotype is identical to the dominant oncogene.

Because the $\mathrm{X} m r k$ oncogene is the molecular equivalent of the genetically defined $T u$-gene, the question can be asked: Is Xmrk the macromelanophore gene? The available evidence for answering "yes" or "no" is conflicting. On the one hand, fish of the "loss of function" mutation that do not develop melanoma due to an insertion in the $\mathrm{X} m r k$ oncogene (Wittbrodt et al., 1989) have also lost the macromelanophore cell lineage. This would argue that $\mathrm{X} m r k$ is the macromelanophore gene. On the other hand, in a preliminary analysis, several populations of different Xiphophorus species that exhibit macromelanophore patterns have been found to contain only the Xmrk protooncogene, but not a functional oncogene. Interestingly, following the appropriate crossing, these genotypes never develop melanoma indicating that the macromelanophore is specified by a separate locus, while X $m r k$ mediates the capacity for neoplastic transformation. The strict cosegregation without imperceptible recombination of the macromelanophore phenotype and the $\mathrm{X} m r k$ oncogene in those genotypes that give rise to hybrid melanoma has thus to be interpreted in the way that if Xmrk and the macromelanophore gene are indeed separate entities, they have to be linked very, very tightly. Certainly much more work is needed to answer these questions.

\section{DOES Xmrk PLAY A SOLO PART IN TUMORIGENESIS OF XIPHOPHORUS?}

Although $\mathrm{X} m r k$ is causative for hereditary melanoma in Xiphophorus, other known oncogenes are expressed as well as was shown by Northern blot analyses. Obviously, these oncogenes are adventitiously activated following Xmrk overexpression (Mäueler et al., 1993). Thus, multiple molecular occurrences are instrumental in generating the complete malignant phenotype of melanoma. The sequence of events, however, remains obscure.

It has been hypothesized that the $T u$-locus is responsible for induction of tumors of all histiotypes and different 
etiologies in Xiphophorus (Anders, 1991). In a preliminary analysis of chemically induced tumors, i.e. fibrosarcoma and melanoma, no expression of X $m r k$ could be detected (Mäueler et al., 1993). This indicates that different molecular pathways can lead to a common tumor phenotype and that activation of oncogenes other than Xmrk can cause tumors in Xiphophorus. The availability of molecular tools that have been developed in the past few years will help to elucidate the complex network of events that finally result in malignant tumors.

\section{REFERENCES}

Adam, D., W. Mäueler, and M. Schartl (1991) Transcriptional activation of the melanoma inducing Xmrk oncogene in Xiphophorus. Oncogene, 6:73-80.

Adam, D., N. Dimitrijevic, and M. Schartl (1993) Tumor suppression in Xiphophorus by an accidentally acquired promoter. Science, 259:816-819.

Ahuja, M.R. and F. Anders (1976) A genetic concept of the origin of cancer, based in part upon studies of neoplasms in fishes. Prog. Exp. Tumor Res., 20:380-397.

Anders, A. F. Anders, and K. Klinke (1973) Regulation of gene expression in the Gordon-Kosswig melanoma system I, II. In: Genetics and Mutagenesis of Fish. J.H. Schröder, ed. Springer-Verlag New York, pp. 33-63.

Anders, F., M. Schartl, A. Barnekow, and A. Anders (1984) Xiphophorus as an in vivo model for studies on normal and defective control of oncogenes. Adv. Cancer Res., 42:191-275.

Anders, F. (1990) A biologist's view of human cancer. In: Modern Trends in Human Leukemia 8, XXII-XLV. R. Neth et al., eds Springer Verlag Berlin.

Anders, F. (1991) Contributions of the Gordon-Kosswig Melanoma System to the present concept of neoplasia. Pigment Cell Res., $3: 7-29$.

Atz, J.W. (1962) Effects of hybridization on pigmentation in fishes of the genus Xiphophorus. Zoologica, 47:153-181.

Breider, H. (1952) Über Melanosarkome, Melaninbildung und homologe Zellmechanismen. Strahlentherapie, 88:619-639.

Dynan, W.S. (1986) Promoters for housekeeping genes. Trends Genet., 2:196-197.

Gordon, M. (1927) The genetics of viviparous top-minnow Platypoecilus: The inheritance of two kinds of melanophores. Genetics, 12:253-283.

Gordon, M. (1931) Hereditary basis of melanosis in hybrid fishes. Am. J. Cancer, 15:1495-1519.

Gordon, M. (1958) A genetic concept for the origin of melanomas. Ann. N.Y. Acad. Sci., 71:1213-1222.

Häussler, G. (1928) Über Melanombildungen bei Bastarden von
Xiphophorus maculatus var. rubra. Klin. Wochenschr., 7: 1561-1562.

Ishii, S., F. Imamoto, Y. Yamanashi, K. Toyoshima, and T. Yamamoto (1985) Characterization of the promoter region of the human c-erbB2 proto-oncogene. Proc. Natl. Acad. Sci. U.S.A., 84:4374-4378.

Kallman, K.D. (1970) Different genetic basis of identical pigment patterns in two populations of platyfish, Xiphophorus maculatus. Copeia, 3:472-487.

Kallman, K.D. (1975) The platyfish, Xiphophorus maculatus. In: Handbook of Genetics, 4. R.C. King, ed. Plenum Press New York, pp. 8-132.

Kosswig, C. (1928) Über Kreuzungen zwischen den Teleostiern Xiphophorus helleri und Platypoecilus maculatus. Z. Indukt. Abstammungs.-Vererbungsl., 47:150-158.

Kosswig, C. (1929) Das Gen in fremder Erbmasse. Züchter, 1:152-157. Kosswig, C. (1965) Genetische Grundlagen des Polymorphismus. Zool. Anz., 175:21-50.

Mäueler, W., F. Raulf, and M. Schartl (1988) Expression of proto-oncogenes in embryonic, adult, and transformed tissue of Xiphophorus (Teleostei: Poeciliidae). Oncogene, 2:421-430.

Mäueler, W., A. Schartl, and M. Schartl (1993) Different expression patterns of oncogenes and proto-oncogenes in hereditary and carcinogen-induced tumors of Xiphophorus. Int. J. Cancer, 55:288-296.

Malitschek, B., J. Wittbrodt, P. Fischer, R. Lammers, A. Ullrich, and M. Schartl (1994) Autocrine stimulation of the Xmrk receptor tyrosine kinase in Xiphophorus melanoma cells and identification of a source for the physiological ligand. J. Biol. Chem. 269:10423-10430.

Schartl, M. (1988) A sex chromosomal restriction-fragment-length marker linked to melanoma-determining $T u$ loci in Xiphophorus. Genetics, 119:679-685.

Schartl, M. and R.U. Peter (1988) Progressive growth of fish tumors after transplantation into thymus-aplastic (nu/nu) mice. Cancer Res., 48:741-744.

Winkler, C., J. Wittbrodt, R. Lammers, A. Ullrich, and M. Schartl (1994) Ligand-dependent tumour induction in medakafish embryos by a X $m r k$ receptor tyrosine kinase transgene. Oncogene, 9:1517-1525.

Wittbrodt, J., D. Adam, B. Malitschek, W. Mäueler, F. Raulf, A. Telling, et al. (1989) Novel putative receptor tyrosine kinase encoded by the melanoma-inducing $T u$ locus in Xiphophorus. Nature, 341:415-421.

Wittbrodt, J., R. Lammers, B. Malitschek, A. Ullrich, and M. Schartl (1992) The Xmrk receptor tyrosine kinase is activated in malignant melanoma of Xiphophorus. EMBO J., 11:4239-4246.

Zander, C.D. (1969) Über die Entstehung und Veränderung von Farbmustern in der Gattung Xiphophorus (Pisces). Mitt. Hamburg Zool. Mus. Inst. 66:241-271.

Zechel, C., U. Schleenbecker, A. Anders, and F. Anders (1988) v-erbB related sequences in Xiphophorus that map to melanoma determining Mendelian loci and overexpress in a melanoma cell line. Oncogene, 3:605-617. 